\title{
Construct Validity of a General Training Climate Scale
}

\author{
J. Bruce Tracey \\ Cornell University
}

Michael J. Tews

Cornell University

J. Bruce Tracey is an associate professor of management at Cornell University's School of Hotel Administration. His current research focuses on training and HR program evaluation, employee turnover, leadership, and labor and employment law.

Michael J. Tews is a doctoral student at Cornell University's School of Hotel Administration. His primary research focuses on the factors associated with training effectiveness, particularly design and transfer strategies for enhancing posttraining performance. 


\begin{abstract}
There is growing evidence that an organization's training climate can influence the effectiveness of formal and informal training activities. Unfortunately, there is limited data regarding the psychometric properties of climate measures that have been used in training research. The purpose of this article is to examine the construct validity of a training climate measure. Results from content adequacy, reliability, aggregation, and convergent, discriminant, and criterion-related validity assessments provide support for the measure 's use in diagnostic and theory testing efforts.
\end{abstract}

Keywords: training climate: training effectiveness; construct validity 


\section{Construct Validity of a General Training Climate Scale}

One of the growing areas of research in the training and development field focuses on the nature and types of factors outside formal learning contexts that may influence the acquisition and application of new knowledge and skills (Salas \& Cannon-Bowers, 2001). Although many variables may influence the effectiveness of training and development efforts, an organization's training climate appears to play an important role. For example, previous research has demonstrated that an organization's training climate is instrumental in preparing individuals for formal development activities and achieving desired learning objectives (e.g., Tracey, Hinkin, Tannenbaum, \& Mathieu, 2001) and in helping to ensure that individuals successfully transfer their newly acquired knowledge and skills to the job upon completion of formal training (e.g., Holton, Bates, \& Ruona, 2000; Rouiller \& Goldstein, 1993; Tracey, Tannenbaum, \& Kavanagh, 1995; Thayer \& Teachout, 1995).

The mounting evidence regarding the relevance of the work environment, and training climate in particular, has shifted attention toward broader and more integrative models of training effectiveness. For example, Kozlowski and Salas (1997) presented a multilevel, systems model of training implementation and transfer that described the ways in which variables at one level of analysis (e.g., work environment) may influence or interact with variables at other levels of analysis (e.g., individual motivation to attend training). Research based on this and related models has generated some needed insight regarding work-related factors that may influence training success and failure; however, theoretical and measurement ambiguities exist. In particular, and as Kozlowski and Salas concluded, "The conceptualization of 'supporting work environment' has not been seriously addressed" (p. 257) within the training literature. Although the nature and relevance 
of constructs such as "perceived organizational support" have been established and linked to a variety of work-related attitudes and outcomes (e.g., D. G. Allen, Shore, \& Griffeth, 2003), focused attention should be given to the conceptual meaning and operationalization of constructs associated with the work environment that are specific to training.

Accordingly, the purpose of this article is to provide some conceptual clarity regarding the training climate construct and present validity evidence for a measure that has been and continues to be used in training climate research. Specifically, we will examine the theoretical foundation and psychometric properties of the scale developed by Tracey and his colleagues, currently labeled General Training Climate Scale (GTCS; Tracey, 1998; Tracey et ah, 1995, 2001). Although previous studies have discussed the conceptual basis for this measure and presented some promising, albeit preliminary results, a comprehensive validity assessment is required to more fully ascertain the utility of this measure.

The current research is important for two reasons. From a practical standpoint, sound measures of the work environment are important for diagnostic purposes. Consider the training needs assessment process. It would be unwise to implement new training programs if the work environment does not adequately prepare trainees for the learning process or support the use of newly acquired knowledge and skills on the job when trainees return to their jobs. As such, valid measures of climate are necessary for clearly identifying potential obstacles to training success. If such obstacles are revealed, then efforts can be taken to address areas of concern prior to making investments in training design and implementation.

From a theoretical perspective, valid measures are critical for advancing models that explain training effectiveness. For example, in addition to influencing the individual (or horizontal) transfer of training process, training climate may also play a role in the vertical transfer 
process - the extent to which individual-level training outcomes become manifest as team- and organization-level outcomes. Based on Kozlowski and Salas's (1997) systems model of transfer, it can be argued that training climate may not only influence individual performance improvements that are due to training but also the degree to which such improvements become institutionalized and reflected in unit- level performance measures (e.g., increased sales, lower employee turnover, improved customer satisfaction, etc.). Thus, training climate may play a critical role in realizing broader changes that result from training and development efforts. However, to fully examine this and related propositions, sound measures and rigorous research methods become sine qua non. Although it can be argued that validation studies may not have the same level of impact as theory development and theory testing, such efforts are necessary and can have a substantial influence on future research. Given the continued interest in the work environment as it pertains to training preparation, performance, and transfer, a rigorous validation analysis of climate measures appears warranted.

We begin by presenting an overview of the climate literature and research that has examined the training climate construct. We then outline our validation strategy and present findings to support the validity of the GTCS. Finally, we discuss the implications of our results for theory and practice.

\section{Organizational Climate}

Climate has a fairly long history in the study of organizations. McGregor (1960) provided one of the earliest conceptualizations of climate and defined the construct as the "day-by-day behavior of the immediate supervisor and of other significant people in the managerial organization" (p. 133). Litwin and Stringer's (1968) book was the first to demonstrate the empirical relevance of the climate construct. They presented results from a number of 
experimental and field studies that showed that different types of climate were related to a variety of individual and organizational outcome variables (e.g., a democratic-friendly business climate resulted in higher satisfaction, whereas an achievement-oriented climate resulted in higher performance).

As research on this topic progressed, additional conceptualizations were offered (e.g., Campbell, Dunnette, Lawler, \& Weick, 1970; Schneider \& Hall, 1972), followed by insightful critiques (e.g., Guion, 1973; James \& Jones, 1974). To date, there is some consensus regarding the definition and conceptual underpinnings of organizational climate. One of the most widely cited conceptualizations was developed by Schneider $(1985,1990)$, who defined climate as the shared perceptions of employees concerning the practices, procedures, and behaviors that get rewarded and supported in a work setting. Moreover, Schneider $(1975,1985)$ suggested that to understand the role of climate in organizations, specific dimensions of climate should be examined. He argued that the context of the appropriate perceptual domain should not be driven by an effort to identify a universal or generic construct that may be applicable to all situations. Rather, climate should be considered as a much broader, multidimensional perceptual variable, and specific dimensions or factor definitions should be determined by a specific criterion or criteria of interest.

The utility of specific and criterion-focused climate constructs has received substantial empirical support. For example, whereas Pritchard and Karasick (1973) failed to demonstrate a link between general climate perceptions and ratings of job-specific performance, Zohar (1980) found that perceptions of safety climate were linked to measures of accident prevention and safety effectiveness. Furthermore, Schneider and his colleagues have demonstrated that employee perceptions of service climate are significantly related to customer perceptions of service quality (e.g., Schneider, Parkington, \& Buxton, 1980; Schneider, White, \& Paul, 1998). Thus, to more 
fully understand the relationship between climate and various individual and organizational phenomena, it appears that climate constructs should be narrowly defined and operationalized and be clearly linked to a study's underlying theoretical foundation and dependent variables of interest.

\section{Training Climate}

Kozlowski and Hults (1987) conducted one of the first studies that examined the relevance and influence of a training-specific climate dimension. They found strong relationships between seven dimensions of "technical updating climate" (e.g., supervisory support, innovation policies, and job assignments) and individual performance, organizational commitment, and growth satisfaction, among other outcomes. Of particular note was the strong relationship between perceptions of technical updating climate and a measure of participation in technical updating activities, measured as the number of hours spent in continuing education seminars, training programs, and other activities related to knowledge and skill acquisition. This study was one of the first to show that consideration should be given the training-specific dimensions of the work environment to explain the success or failure of individual professional development efforts. Unfortunately, little information regarding the construct validity of this climate measure was presented, and additional research on the updating climate dimension has apparently not been pursued.

Subsequent research on training climate became more focused. Rouiller and Goldstein (1993) examined the influence of an organization's transfer climate, defined as "situations and consequences that either inhibit or help to facilitate the transfer of what has been learned in training into the job situation" (p. 379). This particular climate construct was conceptualized to include two distinct factors - situational cues and consequences - with four subscales associated with each factor. Situational cues included goal cues (cues remind trainees to use trained skills), 
social cues (behavior and influence cues exhibited by supervisors, peers, and subordinates), and task and structural cues (cues relating to the design and the nature of the job itself). Consequences included positive and negative feedback and rewards. Although Rouiller and Goldstein showed that the two transfer climate factors each accounted for unique variance in posttraining behavior, the proposed dimensionality of the climate measure was not supported in subsequent research (e.g., Holton, Bates, Seyler, \& Carvalho, 1997).

One likely reason for the lack of empirical distinctiveness among the proposed transfer climate dimensions may be due to the conceptual overlap among the various subscales. For example, it may be quite difficult to distinguish between the support one receives from a supervisor regarding the importance of transferring newly acquired knowledge and skills to the job (i.e., one aspect of the social cues subscale) and the positive feedback received from one's supervisor regarding the effectiveness of one's efforts to transfer what was learned in training to the job (i.e., one aspect of the positive feedback subscale). As such, additional detail regarding the conceptualization of the transfer climate construct was required.

In an effort to provide some of the needed clarity, Holton and his colleagues (Holton et al., 1997, 2000) extended Rouiller and Goldstein's (1993) work and developed the Learning Transfer System Inventory (LTSI). This measure was designed to assess "all factors in the person, training, and organization that influence transfer of learning to job performance" (Holton et ah, 2000, pp. 335-336). The conceptual foundation for this measure was based on previous research that has examined a host of variables that may influence transfer process, such as program content and design, individual characteristics, and features of the work environment-including the transfer climate.

If individuals share perceptions about the work environment, then the climate construct 
embedded within the LTSI may be consistent with Schneider's (1990) aggregate-level conceptualization. On the other hand, a lack of consensus may suggest that the focal construct does not exist at an aggregate level of analysis and, instead, may be consistent with the individual-level or psychological climate construct advanced by James and his colleagues (e.g., James, Jones, \& Ashe, 1990). Alternatively, if individuals do not share perceptions about work environment, then it may be argued that a "weak" climate may exist (e.g., Schneider, Salvaggio, \& Subirats, 2002). The operationalization of the LTSI is based on individual perceptions about the transfer system. Unfortunately, Holton et al. were not explicit about the appropriate level of analysis for their climate construct. Therefore, because the operationalization of the LTSI is based on individual perceptions about the transfer system, we conclude that the transfer climate component of the LTSI is akin to psychological climate, rather than a shared or consensus-based phenomenon.

Using a series of exploratory factor analyses of the 112 items included in the LTSI, 36 items were purported to measure five dimensions of a second-order transfer climate factor: transfer effort-performance expectancies, performance-outcome expectancies, openness to change, performance self-efficacy, and performance feedback. Holton et al. (2000) argued that these climate dimensions fit within work environment domain of the transfer of training model offered by Baldwin and Ford (1988). A close inspection of these dimensions and the associated items lends speculation regarding this classification. Two of the dimensions-openness to change and performance feedback - appear to be perceptual measures of the work environment and may indeed reflect one or two types of climate (and as noted above, most likely psychological climate). However, the remaining three dimensions-transfer effort-performance expectancies, performance-outcome expectancies, and performance self-efficacy - appear to be individual motivation constructs. If climate is a perceptual variable about environmental features, then the 
rationale for including these latter three dimensions in a second- order climate factor may be untenable. Despite this concern, Holton and his colleagues have demonstrated adequate psychometric and predictive validity results across diverse settings (e.g., Holton, Chen, \& Naquin, 2003). Therefore, we believe that the LTSI can be used with confidence as a diagnostic tool, as well as provide a basis for testing and developing new theories about the transfer of training process.

At the same time Holton and his colleagues (1997, 2000) were developing the LTSI, Tracey and his colleagues (Tracey, 1998; Tracey et al., 1995, 2001) were developing another measure of the work environment that complements the aforementioned climate measures and may have somewhat broader applicability. Tracey's measure has two distinctive features. First, whereas Holton et al.'s measure focuses on the transfer of training process with its emphasis on individual-level motivational constructs, Tracey's measure provides a means for linking perceptions about the work environment to training preparation and learning outcomes, as well as the transfer of learned knowledge and skills to the job. Second, Tracey's measure explicitly operationalizes climate as a shared, aggregate-level construct. As such, Tracey's measure provides a direct means for examining multilevel relationships between the variables that have been articulated in current models of training effectiveness (e.g., Noe \& Colquitt, 2002). The following section will elaborate on the conceptual foundation and initial item development of this measure, and subsequent sections will present the results of the current study that lend support for the scale's construct validity.

\section{GTCS}

Tracey et al.’s (1995) initial measure was developed using a deductive scaledevelopment process. Based on reviews of the climate, culture, and training literatures, as well as interviews 
with and observations of respondents included in the 1995 study, 24 items were developed and categorized into the following five dimensions: job challenge ( 5 items), supervisory support (5 items), peer support (5 items), managerial policies and procedures (4 items), and overall continuous learning culture (5 items). These dimensions are similar to those identified in Dubin's (1990) conceptual work on updating and continuous learning, as well as the research by Kozlowski and Hults (1987). At this time, the measure was labeled "continuous learning culture."

However, the results from a series of factor analyses of the 1995 data failed to support the five-factor model; instead, a three-factor model emerged. For example, the supervisory and peer support items loaded on one "social support" factor. In addition, several items cross-loaded on multiple dimensions. Based on these results, subsequent research (Tracey, 1998; Tracey et ah, 2001) refined the conceptualization and operationalization of this measure. It became clear that the focal construct was more closely associated with more observable features of the work environment, compared to less salient elements of organizational settings that are typically associated with cultural phenomena (e.g., Schneider, 1990). It also appeared that the three underlying dimensions identified in the 1995 study were consistent with the propositions set forth in diagnostic theories of organizations (e.g., Nadler \& Tushman, 1980; Daft, 1983), which characterize work contexts in terms of three interrelated systems: social, job- related/technical, and organization. As such, some of the items in the original were dropped because there were not deemed to be consistent with the revised framework. For example, it was determined that the item "This corporation is highly innovative" did not provide a direct indication of an organization's support for learning and development and was, thus, deleted from the scale.

The current conceptualization of training climate is defined as the perceived support from management, work, and the organization for formal and informal training and development 
activities. Based on this conceptualization and previous empirical results, 15 items were retained or modified from the initial 24-item pool to represent three underlying training climate dimensions. The first dimension is managerial support, which is a central part of an organization's social system. This aspect of the work environment reflects the extent to which supervisors and managers encourage on-the- job learning, innovation, and skill acquisition and provide recognition to employees in support of these activities. Bosses can send clear signals regarding the role and value of training, development, and professional growth in the firm, which may, in turn, motivate employee behavior regarding development activities. A sample item is "Supervisors match associates' needs for personal and professional development with opportunities to attend training." The appendix lists all items included in the three subscales.

The second dimension of training climate is job support, which is part of an organization's job-related/technical system. This subscale represents the degree to which jobs are designed to promote continuous learning and provide flexibility for acquiring new knowledge and skills. Job design may facilitate training and related efforts by signaling their importance, as well as by providing opportunities to experiment and utilize newly acquired knowledge and skills, a finding consistent with the work of Ford, Quinones, Sego, and Sorra (1992). A sample item is "Work assignments include opportunities to learn new techniques and procedures for improving performance."

The final dimension, representing the organizational system, is organizational support. This dimension corresponds to policies, procedures, and practices that demonstrate the importance of training and development efforts, such as reward systems and resources to acquire and apply learned skills. For example, it is unlikely that individuals will utilize new knowledge gained from any type of development activity if the organization's performance evaluation procedures do not 
account for the use of the newly acquired knowledge. Moreover, even if individuals are held accountable, it is unlikely they will demonstrate their new knowledge over time without appropriate incentives. Thus, transfer and subsequent preparation for future development activities is contingent upon the alignment between training activities, performance management procedures, and incentive programs. A sample item is "This organization provides the resources necessary for employees to acquire and use new knowledge and skills."

Although the three dimensions may be conceptually distinct, it can be argued that they may be indicators of a more general training climate construct. For example, if employees' jobs are conducive to innovation, learning, and skill development, it is also likely that their supervisors support such activities. It may be difficult if not impossible to divorce the job from a manager's support for various job-related activities. As such, support for learning, training, and related development activities may be a more general phenomenon. However, a more comprehensive validation assessment will provide additional insights regarding this issue.

We should also note that the conceptual foundation for the GTCS is similar to, but distinct from, other constructs associated with perceptions about the work environment. For example, the initial conceptualization relied on previous research regarding the importance of a continuous learning culture for positive training transfer (e.g., Dubin, 1990). Indeed, the current operationalization includes items that address ongoing, continuous efforts to develop new knowledge and skills (e.g., "gaining new information about ways to perform work more effectively is important in this organization"). However, as noted above, the GTCS is not a measure of culture, which is a more deeply embedded organizational phenomenon that is less salient, and perhaps less knowable, than climate. In addition, continuous learning culture reflects consensus about a wider range of work-related phenomena (e.g., learning from mistakes, 
environmental scanning, etc.) that go well beyond the formal and informal development activities that characterize the training climate construct.

It is also evident that the general training climate construct is related to organizational learning. According to Tannenbaum (1997), organizational learning can be defined as "a change in an organization's capacity for doing something new" (p. 438). Such change may be the result of formal interventions designed to enhance employee knowledge and skills (e.g., training). However, organizational learning is a much broader construct and extends well beyond training. For example, organizations may modify their operational practices to avoid a crisis based on learning from past experience. In addition, firms may revise their business strategy in light of new information about competitors. Thus, training climate may be part of, but does not fully define, organizational learning.

\section{Establishing Construct Validity}

To establish the construct validity of the GTCS, we followed the procedures proposed by Cronbach and Meehl (1955) and Schwab (1980). We will begin by presenting the results from a study that examined the content adequacy (i.e., content validity). We will then present the results from a second study that assessed the other major requirements for establishing construct validity: reliability (internal consistency), convergent and discriminant validity, and criterion-related validity. This study will also examine the extent to which individual ratings of training climate may be aggregated to represent a higher level construct.

\section{Study 1}

An often overlooked yet critical step in the scale development process is an assessment of content adequacy, or content validity. This assessment allows for the deletion of items that may be conceptually inconsistent with the focal construct(s). Several content adequacy assessment 
methods have been described in the research methods literature (e.g., Nunnally, 1978; Nunnally \& Bernstein, 1994). One common method requires respondents to categorize, or sort, items based on their similarity to construct definitions. Naive respondents are presented with construct definitions without titles and are asked to match items with a corresponding definition. An agreement index is computed and compared to a threshold or standard that is identified prior to administration of the sorting task.

A recently developed method for conducting content adequacy assessments was presented by Hinkin and Tracey (1999). This process is similar to sorting techniques but utilizes a rating process and analysis of variance for determining item retention. There are three primary benefits of this procedure. First, small sample sizes (e.g., $n=30$ to 50) can be used. This is advantageous both because of convenience and also for statistical purposes (i.e., significant findings have more practical meaning). Second, the process requires only that respondents are not biased and possess sufficient intellectual ability to perform the item rating tasks. Finally, the analytical procedure is based on an analysis of variance technique, which reduces the use of subjective judgment for item retention. The specific procedures used for this assessment are described below.

\section{Method}

\section{Sample and procedures.}

The sample for this study consisted of 32 graduate business students at a large, private university located in the northeastern United States. The respondents participated on a voluntary and anonymous basis. The average age was 28 years, $40 \%$ were female, and the average work experience was about 6 years.

A content adequacy survey was developed such that the definition of one of the three training climate dimensions was presented at the top of each page of the survey, followed by a 
random listing of the 15 training climate items. Three versions of the survey were then administered, each with the definitions presented in a different order to control for response bias that may occur from order effects. Respondents rated each of the 15 training climate items on the extent to which they believed that the items were consistent with each of the three training climate dimensions. Response choice alternatives ranged from 1 (not at all) to 5 (completely). No statistically significant differences among the responses across the versions were found.

\section{Results}

A series of one-way ANOVAs was conducted to compare an item's mean rating on one dimension to the item's ratings on the other dimensions. This approach provides a basis for determining whether an item's mean score is statistically significantly higher on the proposed theoretical construct. Duncan's multiple range test was used to address concerns regarding Type I error rates by holding the probability of making a Type I error for the entire set of comparisons to an a priori defined alpha.

The results from the ANOVAs and Duncan's multiple comparison tests indicated that 14 out of 15 of the items were judged to be consistent with the proposed dimension. $F$ ratios ranged from 9.55 to $46.62(d f=2,93 ; p<.001)$. The one exception was for the item, "Supervisors match associates' needs for personal and professional development with opportunities to attend training." The mean rating for this item was higher for the purported dimension (managerial support, 3.44) compared to ratings for the other dimensions (job support, 3.31; organizational support, 3.28); however, the $F$ ratio was less than one and nonsignificant.

\section{Study 2}

For this study, we gathered data from a sample of restaurant managers to assess the remaining indicators of construct validity. Given the high degree of daily involvement in unit 
operations, we contend that restaurant managers can provide a valid source of information regarding the training climate of each unit. The sample and data collection procedures are described in detail below.

\section{Convergent validity.}

To assess convergent validity, we used a procedure that is consistent with previous validation efforts (e.g., Sturman \& Short, 2000) in which a series of factor analyses were conducted to examine the proposed dimensionality of the GTCS. First, the data were subjected to a principal components analysis. Then, we conducted a series of confirmatory factor analyses in which we compared the fit statistics for the proposed three-factor model to the fit statistics of a one-factor model. A one-factor model was selected as the comparative referent based on previous research that collapsed the three climate scales into a single general climate indicator (e.g., Tracey et al., 2001).

It should be noted that whereas it is preferable to conduct exploratory and confirmatory factor analyses using independent samples, the use of both exploratory and confirmatory factor analysis can yield distinctive insights regarding the dimensionality of the focal scales.

\section{Discriminant validity.}

Similar to the procedure for examining convergent validity, discriminant validity was assessed using a series of exploratory and confirmatory factor analyses to examine the extent to which the items associated with the GTCS loaded uniquely on the proposed factors, in comparison to items that assess distinct but related constructs. For this assessment, we compared the GTCS to the global service climate scale developed by Schneider et al. (1998) and the affective commitment scale developed by N. J. Allen and Meyer (1990).

As noted above, it has been argued that climate is a multidimensional construct and that 
multiple climates may exists within a specific context. The case for discriminant validity of the GTCS could be made if it is found to be empirically distinct from an established climate measure, particularly in a setting in which both training and service climate have a great deal of strategic and operational relevance (i.e., a restaurant company that implements ongoing service training programs to ensure consistent and high quality customer service). In addition, because previous research has shown that the GTCS is significantly related to organizational commitment (e.g., Tracey et al., 2001), comparisons among the GTCS, service climate, and organizational commitment scales should provide a fairly robust assessment of the empirical distinctiveness and practical utility of the focal measure.

First, we conducted principal components analyses of the GTCS items and the items associated with each of the comparison scales (i.e., separate analyses were conducted for each of the comparison measures). Then, we conducted confirmatory factor analyses to examine the fit of a four-factor model in which the GTCS items were specified to load on the three proposed dimensions and the items for the comparison scales were specified to load on distinct factors. Again, separate analyses were conducted for each of the comparison scales.

\section{Reliability.}

For this assessment, we examined the internal consistency of the GTCS. A value of .70 is considered acceptable, with values above .90 ultimately desirable (Nunnally \& Bernstein, 1994).

\section{Aggregation.}

Consistent with previous research, we examined the extent to which the GTCS may reflect an aggregate level phenomenon. Although climate may exist at many levels of analysis (e.g., James et al., 1990), we focused on the business unit level (i.e., restaurant/store level) because it is the most ubiquitous operational element of the sponsoring organization for our study. Each store is 
independently operated by a management team that is fully accountable for financial performance and service quality, among other factors that are critical for success in the restaurant industry. As such, managers are inextricably involved in the day-to-day operations of each restaurant. Given the high level of interpersonal contact among managers and a fairly limited number of supervisory and line staff, ${ }^{1}$ it is likely that perceptions about phenomena such as climate would be shared among most individuals within this type of work setting and thus become manifest at the unit level. For this assessment, we utilized James, Demaree, and Wolf's (1984) within-group interrater agreement index.

\section{Criterion-related validity.}

Although previous research has provided some evidence regarding the criterion-related validity of the GTCS (e.g., Tracey et al., 2001), additional support is warranted. For the current study, we examined the relationship between training climate and a unit-level measure of training investment. It can be argued that a positive training climate should be associated with the extent to which organizations invest in formal training activities. That is, perceptual indicators of training climate should coincide with objective indicators of the value of training. Therefore, we should find a positive relationship between training climate and the amount of hours that an organization or business unit dedicates to formal training activities.

\section{Method}

Sample and procedures. The data for this part of our study came from managers who worked for a company that owns approximately 120 casual-theme, midscale restaurants throughout the United States. The average number of seats per restaurant is about 175 . The organization also has about 80 franchised stores; however, the data for the current study was obtained only from the corporate-owned units. As part of a larger study on employee opinions, 
surveys were administered via mail to all unit managers. An accompanying cover letter explained the general nature of our study, guaranteed confidentiality of responses, and instructed the respondents to return their completed survey (using an accompanying self-addressed, postage-paid envelope) directly to the authors. Of the approximately 400 surveys that were distributed, 246 complete and useable surveys from were returned, yielding a response rate of approximately $62 \%$. The average age of the respondents was about 35 years, $20 \%$ were female, and the average respondent had worked for the organization for about 3.5 years.

\section{Measures.}

In addition to the GTCS, responses to the following measures were gathered:

Service climate: As noted above, service climate was assessed using Schneider et al.'s (1998) global service climate measure. This seven-item scale was developed for a banking sample, so slight wording modifications were made to accommodate the context used in this study. A sample item was "How would you rate the knowledge and skills of employees to deliver superior quality work and service?" Response choice alternatives ranged from 1 (very poor) to 5 (excellent).

Organizational commitment: The affective commitment scale developed by N. J. Allen and Meyer (1990) was used to assess organizational commitment. A sample item was "I feel emotionally attached to this organization." Response choice alternatives ranged from 1 (strongly disagree) to 5 (strongly agree).

Training investment:One way to assess training investment is to examine the number of hours employees participate in formal development activities. For this study, the sponsoring organization tracked the number of hours that each unit invests in new employee skill development (via a payroll function). It should be 
emphasized that this measure reflects only one type of training investment made by the sponsoring organization, which offers a number of training and development opportunities to all employees throughout their tenure with the organization.

The content of the new employee training was broad, with topics ranging from policies and procedures to job-specific tasks, duties, and responsibilities. This measure was made available to the authors for the month immediately alter the survey data were collected and reflects the ratio of the number of training hours per new employee trained during the focal month. Managers have considerable discretion regarding the amount and type of training that is provided to new employees. This discretion is based primarily on the variability in the capabilities and experience of those who apply for and are ultimately selected for line-level positions, as well as the specific needs of the restaurant. For example, in some locations, competition is quite high. As such, both service quality and food quality are extremely important. Thus, managers in this type of context may have to spend considerably more time and effort training new employees to meet and exceed standards to generate adequate revenues and maintain market share.

We also obtained employee turnover data (voluntary and involuntary, combined) from each restaurant unit for the month in which the survey data were gathered to serve as a control variable. It should be noted that there was no relationship between the size of the restaurant (in terms of the number of seats) and turnover.

\section{Results}

Convergent validity. For this analysis, we used an oblique rotation and a principal components method of extraction. A scree test and an eigenvalue cutoff of 1.0 or higher were used to select the number of factors, and items with factor loadings of .40 or higher on only one factor were used to define the factor. The results yielded a three- factor solution that accounted for $65.8 \%$ 
of the variance. All items loaded exclusively on the proposed factor. Factor loadings are reported in Table 1.

\section{Insert Table 1}

For the confirmatory factor analysis, model fit was evaluated using the sample variance-covariance matrix of the 15 items as input and a maximum likelihood solution. For the three-factor model, the overall chi-square was statistically significant $\left(\mathrm{X}^{2}=229.53, d f=87, p<\right.$ .01 ), the Comparative Fit Index was .97, the Tucker-Lewis Index (NNFI) was .96, and the standardized root mean square residual was .048. Based on Hu and Bender's (1999) cutoff criteria for fit indexes, these results support the proposed dimensionality of the training climate measure. All factor item loadings were statistically significant $(\mathrm{p}<.01)$ and ranged from .66 to .85 . The factor correlations were as follows: .65 between organizational support and job support, .42 between organizational support and managerial support, and .60 between job support and managerial support.

For the one-factor model, the overall chi-square was statistically significant $\left(\mathrm{X}^{2}=761.51\right.$, $d f=90, p<.01$ ), the Comparative Fit Index was .84, the NNFI was .81 , and the standardized root mean square residual was .119. These results, as well as a chi-square difference test that compared the fit of the two models, show that the three-factor model was superior to the one-factor model. Table 2 presents the fit statistics from the confirmatory factor analyses of the proposed and alternative models. Table 3 presents the factor loadings of the confirmatory factor analysis of the GTCS items (three-factor model only).

\section{Discriminant validity.}

Similar to the procedures described above, we first used an oblique rotation and a principal components method of extraction (and the same criteria for factor and item retention) to assess the 
dimensionality of the

\section{Insert Table 2}

\section{Insert Table 3}

GTCS items and the items for the two comparison scales. For the GTCS and service climate items, the results generated a four-factor model that accounted for $61.8 \%$ of the variance. All but one item loaded exclusively on the proposed factor. The service climate item "tools, technology, and other resources provided to employees to support the delivery of superior quality work and service" loaded with the organizational support items from the GTCS. Factor loadings are reported in Table 4.

The principal components analysis of the GTCS and organizational commitment items yielded a five-factor solution that accounted for $68.4 \%$ of the variance. In this case, all GTCS items loaded exclusively on the proposed factor, and the organizational commitment items loaded on two separate factors. Factor loadings are reported in Table 5.

For the confirmatory factor analysis of the four-factor model of the GTCS and general climate items, the overall chi-square was statistically significant $\left(\mathrm{X}^{2}=490.30, d f=203, p<.01\right)$, the Comparative Fit Index was .96 , the NNFI was .95 , and the standardized root mean square residual was .066. All factor item loadings were statistically significant $(p<.01)$ and ranged from .67 to .85 for the GTCS items and from .44 to .79 for the service climate items. The factor correlations between the scales were as follows; .64 between organizational support and job 
support, .42 between organizational support and managerial support, .60 between job support and managerial support, .42 between organizational support and service climate, .49 between managerial support and service climate, and .62 between managerial support and service climate.

\section{Insert Table 4}

For the confirmatory factor analysis of the four-factor model of the GTCS and organizational commitment items, the overall chi-square was statistically significant $\left(\mathrm{X}^{2}=473.56\right.$, $d f$ - 203, $p<.01$ ), the Comparative Fit Index was .96, the NNFI was .96, and the standardized root mean square residual was .053. All factor item loadings were statistically significant $\{p<.01)$ and ranged from .66 to .85 for the GTCS items and from -.41 to .86 for the commitment items. The factor correlations between the scales were as follows: .65 between organizational support and job support, .42 between organizational support and managerial support, .60 between job support and managerial support, .53 between organizational support and commitment, .51 between managerial support and commitment, and .40 between managerial support and commitment.

Table 2 presents the fit statistics from the confirmatory factor analyses conducted for the discriminant validity assessment. Tables 6 and 7 present the factor loadings from the confirmatory factor analyses of the GTCS items and the two comparison measures.

\section{Insert Table 5}

\section{Reliability.}

Consistent with the convergent and discriminant validity findings, the results from the reliability analysis showed that Cronbach's alpha was .85 for job support dimension, .87 for the managerial support dimension, and .87 for the organizational support dimension. 


\section{Aggregation.}

To make the case for unit-level aggregation, we first identified the units in which we had at least three complete surveys. Thirty-eight units met this criterion $(N=124$ respondents), and the results showed that the average within-group interrater agreement index was .94. The index range was .63 to .99 , and the average number of respondents per unit was just greater than three.

\section{Criterion-related validity.}

For this validity assessment, we first analyzed the correlations between the GTCS dimensions and the training investment variable. Due to the relatively low number of units that had three or more complete survey responses, we used the data from units that had two or more survey responses $(N-84)$. The correlation was $.20(/$ ? $<.05)$ for job support,. $15(p<.10)$ for managerial support, and .06(ns) for organizational support. We also conducted regression analyses to determine if the subscales added any explanatory variance in the training investment variable, beyond that account for by employee turnover. The only significant result was found for the job support scale $\left(R^{2}-.06, F=2.73, d f=83, p=.07\right.$; beta $\left.=.22, p<.05\right)$. The turnover variable was also significant in the equation (beta $=.17, p<.10$ ).

\section{Insert Table 6}

\section{Discussion}

To achieve the maximum benefit from formal and informal development efforts, it is critical to identify forces that may either facilitate or inhibit training success. The results from this study extend previous research and provide evidence for the construct validity of the GTCS. Our findings showed that the GTCS appears to measure distinctive characteristics of the work 
environment and, as such, may provide important information regarding the extent to which training efforts may achieve desired outcomes. By using the GTCS, practitioners may be able to make better decisions regarding when and how to invest in continuous learning activities. Similarly, scholars can incorporate the GTCS and training climate into models that explain processes for enhancing the quality of training preparation, performance, and transfer.

One of the unique features of this study was the use of a content adequacy assessment as the first step in establishing construct validity. The procedures show that the GTCS item pool could be categorized into three conceptually distinct dimensions. In addition, we demonstrated the relative ease by which this type of validity may be assessed. As noted by Hinkin and Tracey (1999), “Assessing evidence of content validity does not necessarily require complicated, cumbersome analytical analyses or huge samples. Rather, the process can be quite straightforward and provide an efficient means for establishing and interpreting the utility of any measure" (p. 175). We hope that others follow this example and consider

\section{Insert Table 7}

content validity assessment as an integral part of the construct validity process.

The results from Study 2 complemented and extended the content validity assessment in several ways. The convergent validity assessment showed that there are three related but distinct training climate dimensions and that the information generated from the GTCS can help identify which aspects of the work environment may need to be modified to insure training success. For example, managers may be quite supportive of learning activities, and the organization may offer 
excellent training programs, but the demands of work may be such that they prevent individuals from utilizing newly acquired knowledge and skills. As such, information from the GTCS can be used to prioritize action steps and, in this case, change the structure of jobs in order to realize the benefits of training.

The discriminant validity assessment not only demonstrated the uniqueness of the GTCS but also showed that multiple dimensions of climate may exist simultaneously within work settings. Future research should account for the multidimensional qualities of climate and carefully consider the distinctions among relevant dimensions when extending and developing new models of training effectiveness. Based on the current study (i.e., correlations between the training and service climate factor scores), it can be argued that an organization's training climate may play an important role in the development and maintenance of a positive service climate, which has been shown to influence customer perceptions of service quality and other outcomes that are critical for service organizations (Schneider \& Bowen, 1995). Thus, the relevance of training climate for achieving results-level outcomes, particularly in service settings, may be broader and more complex that currently considered.

Whereas the aggregation analysis showed that the GTCS can be used as a measure of shared perceptions, it should be noted that in some organizations, there may be substantial variability in the extent to which climate perceptions are shared (e.g., dysfunctional organizations in which there is little cross-functional cooperation, communication, and coordination). However, low within-group interrater agreement does not necessarily mean that the focal construct does not exist. Schneider et al.'s (2002) recent study showed that climate strength, which was operationalized as the degree of within-group variability in climate perceptions, moderated the relationship between employee perceptions about service climate (specific to managerial practices 
that support employee service behaviors) and customer ratings of service quality. By extension, training climate strength may moderate the relationship between training climate perceptions and numerous variables associated with pretraining preparation, performance, and transfer. Thus, climate strength may play an important role in further explaining how and why training results are achieved and may provide a means for extending and refining current models of training effectiveness.

For example, Noe and Colquitt's (2002) model shows that climate, as a general construct, is directly related to pretraining attitudes and motivation, learning outcomes, transfer of training, and job performance. If climate strength moderates the relationships between climate perceptions and variables such as pretraining motivation and transfer behaviors (e.g., the magnitude of the relationship between training climate and posttraining transfer behaviors may be higher when the variance in ratings is low compared to situations in which the variance in training climate ratings is high), then not only do these moderating influences need to be accounted for, but it may be that different dimensions of climate - training and otherwise - have differential effects on the posited relationships. For example, managerial support may be more important for pretraining preparation, whereas job support may be more important for facilitating transfer. As such, it appears that we are just beginning to understand the nature of the influence that training climate may have on training effectiveness.

We should also emphasize that theory and research in fields such as organizational change, business policy and strategy, and labor economics, which have defined several cross-level and multilevel dynamics regarding training and development efforts, should be incorporated into the growing body of training theory and research to provide a more comprehensive framework of factors beyond the immediate learning context that may influence training effectiveness. Many of 
these factors may either shape or be shaped by training climate and, as such, should be integrated within the evolving explanatory frameworks. For example, there is evidence that high-performance work systems (HPWS), which are characterized by abroad range of HR policies, programs, and activities (e.g., highly selective staffing programs, pay-for-performance policies, extensive training and development opportunities, etc.) may influence firm-level performance (e.g., Youndt, Dean, Snell, \& Lepak, 1996). Firms that adopt HPWS may create a stronger and more positive training climate (due to the emphasis on longer term development) than firms which adopt a more efficiency-oriented HR strategy (which tends to emphasize more immediate training needs). If so, then multilevel training theories (e.g., Kozlowski \& Salas, 1997) should account for more strategically oriented factors (e.g., HPWS) which may influence training climate and a host of training-related variables (e.g., training transfer).

Before concluding, we should note a few limitations of the current study. First, as noted above, it would have been preferable to conduct the exploratory and confirmatory factor analyses using separate samples. In addition, we did not provide an opportunity for others (i.e., subject matter experts) to add items to those that were examined in the current study. Thus, the current measure may not capture the entire content domain of the training climate construct. Also, it would have been helpful to include measures of organizational learning, continuous learning culture, and related constructs to further establish the convergent and discriminant validity of the GTCS. And finally, the results from the criterion-related validity study did not fully support the predictive validity of all three training climate dimensions. As such, future research should examine other dependent variables that may be influenced by training climate perceptions.

In sum, research and practice have recognized that training climate plays an important role in training effectiveness. We have provided some conceptual clarity regarding the training climate 
construct and presented evidence regarding the construct validity of the GTCS. The development of the GTCS yields a more complete conceptualization of training climate and provides a useful tool to help examine the impact of the work environment on training effectiveness. We should emphasize, however, that this article represents a first step in the process of demonstrating construct validity of the GTCS. Although our results suggest that studying training climate may promote insights into the effects of the work environment on training effectiveness, it is important to replicate these findings in other organizational settings and continue to integrate training climate into new theories of training and development. We hope that the GTCS will provide a tool to facilitate such research. 
Notes

${ }^{1}$ In each store, there is usually one general manager, one assistant general manager, two to three frontline managers, and approximately 20 full-time-equivalent line staff employees. 
Table 1

Pattern Matrix Factor Loadings From the Exploratory Factor Analysis of the General Training Climate Scale (GTCS)

\begin{tabular}{lccc}
\hline Item $^{\mathrm{a}}$ & Factor 1 & Factor 2 & Factor 3 \\
\hline OS3 & .84 & & \\
OS4 & .81 & & \\
OS1 & .80 & & \\
OS2 & .74 & & \\
OS5 & .73 & .86 & \\
MS1 & & .83 & -.82 \\
MS2 & & .78 & -.81 \\
MS3 & & .77 & -.79 \\
MS4 & & & -.70 \\
MS5 & & & -.66 \\
JS2 & & & \\
JS5 & & & \\
JS4 & & & \\
JS1 & & & \\
JS3 & & & \\
\hline
\end{tabular}

Note. Only factor loadings greater than .20 are presented. OS = organizational support; MS = managerial support; JS = job support.

a. All items correspond in order to those listed in the appendix. 
Table 2

Fit Statistics for Proposed and Alternative Models

\begin{tabular}{lccccc}
\hline Model & $\chi^{2}$ & df & CFI & NNFI & SRMSF \\
\hline $\begin{array}{l}\text { Convergent validity } \\
\quad \text { Proposed three-factor }\end{array}$ & 229.53 & 87 & .97 & .96 & .048 \\
$\quad$ Alternative one-factor & 761.51 & 90 & .84 & .81 & .119 \\
$\begin{array}{l}\text { Discriminant validity } \\
\quad \text { Four-factor with service climate }\end{array}$ & 490.30 & 203 & .96 & .95 & .066 \\
$\quad$ Four-factor with commitment & 473.56 & 203 & .96 & .96 & .053 \\
\hline
\end{tabular}

Note. $\mathrm{CFI}=\mathrm{Comparative} \mathrm{Fit} \mathrm{Index;} \mathrm{NNFI} \mathrm{=} \mathrm{Tucker-Lewis} \mathrm{Index;} \mathrm{SRMSR} \mathrm{=} \mathrm{standardized} \mathrm{root} \mathrm{mean}$ square residual. 
Table 3

Convergent Validity LISREL Estimates of Factor Loadings for the General Training Climate Scale (GTCS)

\begin{tabular}{lccc}
\hline Item & $\begin{array}{c}\text { Managerial Support } \\
\text { (MS) }\end{array}$ & $\begin{array}{c}\text { Job Support } \\
\text { (JS) }\end{array}$ & $\begin{array}{c}\text { Organizational Support } \\
\text { (OS) }\end{array}$ \\
\hline MS1 & .80 & & \\
MS2 & .81 & & \\
MS3 & .74 & & \\
MS4 & .74 & & \\
MS5 & .71 & .76 & \\
JS1 & & .79 & \\
JS2 & & .68 & \\
JS3 & & .69 & \\
JS4 & .75 & \\
JS5 & & \\
OS1 & & .66 \\
OS2 & & & .76 \\
OS3 & & & .85 \\
OS4 & & & .80 \\
OS5 & & & \\
\hline
\end{tabular}




\section{Table 4}

Pattern Matrix Factor Loadings From the Exploratory Factor Analysis of the General Training Climate Scale (GTCS) and General Service Climate Items

\begin{tabular}{|c|c|c|c|c|}
\hline Item & Factor 1 & Factor 2 & Factor 3 & Factor 4 \\
\hline MS2 & .85 & & & \\
\hline MS1 & .84 & & & \\
\hline MS3 & .79 & & & \\
\hline MS4 & .77 & & & \\
\hline MS5 & .70 & & & \\
\hline Os3 & & -.86 & & \\
\hline OS4 & & -.83 & & \\
\hline OS1 & & -.78 & & \\
\hline OS2 & & -.77 & & \\
\hline OS5 & & -.75 & & .27 \\
\hline SC7 & & -.46 & .27 & \\
\hline SC1 & & & .85 & \\
\hline $\mathrm{SC} 4$ & & & .84 & \\
\hline SC5 & .23 & & .68 & \\
\hline SC6 & & & .60 & \\
\hline SC2 & & & .57 & \\
\hline SC3 & & -.25 & .40 & \\
\hline JS5 & & & & .75 \\
\hline JS4 & & & & .75 \\
\hline JS2 & & & & .74 \\
\hline JS1 & & -.26 & & .67 \\
\hline JS3 & & & & .64 \\
\hline
\end{tabular}

Note. Only factor loadings greater than .20 are presented. $M S=$ managerial support; OS = organizational support; SC = service climate; JS = job support. 
Table 5

Pattern Matrix Factor Loadings From the Exploratory Factor Analysis of the General Training Climate Scale (GTCS) and Organizational Commitment Items

\begin{tabular}{|c|c|c|c|c|c|}
\hline $\mathrm{Item}^{7}$ & Factor 1 & Factor 2 & Factor 3 & Factor 4 & Factor 5 \\
\hline OS3 & .83 & & & & \\
\hline OS1 & .80 & & & & \\
\hline OS4 & .74 & & & & -.21 \\
\hline OS2 & .72 & & & & \\
\hline OS5 & .67 & & & & \\
\hline MS1 & & .87 & & & \\
\hline MS2 & & .82 & & & \\
\hline MS3 & & .78 & & & \\
\hline MS4 & & .77 & & & \\
\hline MS5 & & .74 & & & \\
\hline OC2 & & & .91 & & .31 \\
\hline OC3 & & & .82 & & \\
\hline OC6 & & & .66 & & -.31 \\
\hline OC1 & & & .63 & & -.22 \\
\hline JS2 & & & & .83 & \\
\hline JS5 & & & & .82 & \\
\hline JS4 & & & & .78 & \\
\hline JS1 & .23 & & & .69 & \\
\hline JS3 & & & & .65 & \\
\hline OC4 & & & & & .78 \\
\hline OC7 & & & .46 & & -.52 \\
\hline OC5 & & & .38 & & -.50 \\
\hline
\end{tabular}

Note. Only factor loadings greater than .20 are presented. OS = organizational support; $\mathrm{MS}=$ managerial support; $\mathrm{OC}=$ organization commitment; JS = job support. 


\section{Table 6}

Discriminant Validity LISREL Estimates of Factor Loadings for the General Training Climate Scale (GTCS) and Schneider, White, and Paul's (1998) Global Service Climate Measure

\begin{tabular}{|c|c|c|c|c|}
\hline Item & $\begin{array}{c}\text { Managerial } \\
\text { Support } \\
\text { (MS) }\end{array}$ & $\begin{array}{c}\text { Job } \\
\text { Support } \\
\text { (JS) }\end{array}$ & $\begin{array}{l}\text { Organizational } \\
\text { Support } \\
\text { (OS) }\end{array}$ & $\begin{array}{c}\text { Service } \\
\text { Climate } \\
\text { (SC) }\end{array}$ \\
\hline MS1 & .76 & & & \\
\hline MS2 & .75 & & & \\
\hline MS3 & .75 & & & \\
\hline MS4 & .77 & & & \\
\hline MS5 & .79 & & & \\
\hline JS1 & & .77 & & \\
\hline JS2 & & .80 & & \\
\hline JS3 & & .65 & & \\
\hline JS4 & & .66 & & \\
\hline JS5 & & .68 & & \\
\hline OS1 & & & .79 & \\
\hline OS2 & & & .76 & \\
\hline OS3 & & & .81 & \\
\hline OS4 & & & .80 & \\
\hline OS5 & & & .67 & \\
\hline SC1 & & & & .65 \\
\hline SC2 & & & & .72 \\
\hline SC3 & & & & .69 \\
\hline SC4 & & & & .64 \\
\hline SC5 & & & & .63 \\
\hline SC6 & & & & .53 \\
\hline SC7 & & & & .55 \\
\hline
\end{tabular}


Table 7

Discriminant Validity LISREL Estimates of Factor Loadings for the General Training Climate Scale (GTCS) and Organizational Commitment Scale Items

\begin{tabular}{|c|c|c|c|c|}
\hline Item & $\begin{array}{c}\text { Managerial } \\
\text { Support } \\
\text { (MS) }\end{array}$ & $\begin{array}{c}\text { Job } \\
\text { Support } \\
\text { (JS) }\end{array}$ & $\begin{array}{c}\text { Organizational } \\
\text { Support } \\
\text { (OS) }\end{array}$ & $\begin{array}{c}\text { Organizational } \\
\text { Commitment } \\
\text { (OC) }\end{array}$ \\
\hline MS1 & .80 & & & \\
\hline MS2 & .81 & & & \\
\hline MS3 & .74 & & & \\
\hline MS4 & .73 & & & \\
\hline MS5 & .71 & & & \\
\hline JS1 & & .77 & & \\
\hline JS2 & & .79 & & \\
\hline JS3 & & .68 & & \\
\hline JS4 & & .69 & & \\
\hline JS5 & & .75 & & \\
\hline OS1 & & & .66 & \\
\hline OS2 & & & .69 & \\
\hline OS3 & & & .76 & \\
\hline OS4 & & & .84 & \\
\hline OS5 & & & .80 & \\
\hline OC1 & & & & .72 \\
\hline OC2 & & & & .59 \\
\hline OC3 & & & & .80 \\
\hline OC4 & & & & -.40 \\
\hline OC5 & & & & .75 \\
\hline OC6 & & & & .86 \\
\hline OC7 & & & & .70 \\
\hline
\end{tabular}




\section{References}

Allen, D. G., Shore, L. M., \& Griffeth, R. W. (2003). The role of perceived organizational support and supportive human resource practices in the turnover process. Journal of Management, 29, 99-118.

Allen, N. J., \& Meyer, J. R (1990). The measurement and antecedents of affective, continuance, and normative commitment. Journal of Occupational Psychology, 63, 1-18.

Baldwin, T. T, \& Ford, J. K. (1988). Transfer in training: A review and directions for future research. Personnel Psychology, 41, 63-105.

Campbell, J. P., Dunnette, M. D., Lawler, E. E., \& Weick, K. E. (1970). Managerial behavior, performance, and effectiveness. New York: McGraw-Hill.

Cronbach, L. J., \& Meehl, P. E. (1955). Construct validity in psychological tests. Psychological Bulletin, 52, 281-30

Daft, R. L. (1983). Organization theory and design. St. Paul, MN: West.

Dubin, S. S. (1990). Maintaining competence through updating. In S. L. Willis \& S. S. Dubin (Eds.), Maintaining professional competence (pp. 9-43). San Francisco: Jossey-Bass.

Ford, J. K., Quinones, M. A., Sego, D. J., \& Sorra, J. S. (1992). Factors affecting the opportunity to perform trained tasks on the job. Personnel Psychology, 45, 511-527.

Guion, R. M. (1973). A note on organizational climate. Organizational Behavior and Human Performance, 9, 120-125.

Hinkin, T. R., \& Tracey, J. B. (1999). An analysis of variance approach to content validation. Organizational Research Methods, 2, 175-186.

Holton, E. F., Bates, R. A., \& Ruona, W. E. A. (2000). Development of a generalized learning transfer system inventory. Human Resource Development Quarterly, 11, 333-360. 
Holton, E. F., Bates, R. A., Seyler, D., \& Carvalho, M. (1997). Toward a construct validation of a transfer climate instrument. Human Resource Development Quarterly, 8, 95-113.

Holton, E. R, Chen, J., \& Naquin, S. S. (2003). An examination of learning transfer system characteristics across organizational settings. Human Resource Development Quarterly, 14, 459-482.

Hu, L., \& Bentler, P. M. (1999). Cutoff criteria for fit indexes in covariance structure analysis: Conventional criteria versus new alternatives. Structural Equations Modeling, 6, 1-55.

James, L. R., Demaree, R. G., \& Wolf, G. (1984). Estimating within-group inter-rater reliability with and without response bias. Journal of Applied Psychology, 69, 85-98.

James, L. R., \& Jones, A. R (1974). Organizational climate: A review of theory and research. Psychological Bulletin, 81, 1096-1 I 12.

James, L. R., Jones, A. R, \& Ashe, D. K. (1990). The meaning of organizations: The role of cognition and values. In B. Schneider (Ed.), Organizational climate and culture. San Francisco: Jossey-Bass.

Ko/.lowski, S. W, \& Hults, B. M. (1987). An exploration of climates for technical updating and performance. Personnel Psychology, 40, 539-563.

Kozlowski, S. W., \& Salas, E. (1997). A multilevel organizational systems approach for the implementation and transfer of training. In J. K. Ford, S. W. Kozlowski, K. Kraiger, E. Salas, \& M. S. Teachout (Eds.), Training effectiveness in work organizations. Mahwah, NJ: Lawrence Erlbaum.

Litwin, G., \& Stringer, R. (1968). Motivation and organizational climate. Cambridge, MA: Flarvard University Press.

McGregor, D. M. (1960). The human side of enterprise. New York: McGraw-Hill. 
Nadler, S., \& Tushman, M. (1980). A model for diagnosing organizational behavior. Organizational Dynamics, 9, 35-51.

Noe, R. A., \& Colquitt, J. A. (2002). Planning for training impact: Principles of training effectiveness. In K. Kraiger (Ed.), Creating, implementing, and managing effective training and development: State-of-the-art lessons for practice (pp. 53-79). San Francisco: Jossey-Bass.

Nunnally, J. C. (1978). Psychometric theory. New York: McGraw-Hill.

Nunnally, J. C., \& Bernstein, I. J. (1994). Psychometric theory. New York: McGraw-Hill.

Pritchard, R. D., \& Karasick, B. W. (1973). The effect of organizational climate on managerial job performance and job satisfaction. Organizational Behavior and Human Performance, 9, $126-146$.

Rouiller, J. A., \& Goldstein, I. L. (1993). The relationship between organizational transfer climate and positive transfer of training. Human Resource Development Quarterly, 4, 377390.

Salas, E., \& Cannon-Bowers, J. A. (2001). The science of training: A decade of progress. Annual Review of Psychology, 52, 471-499.

Schneider, B. (1975). Organizational climates: An essay. Personnel Psychology, 36, 447-479.

Schneider, B. (1985). Organizational behavior. Annual Review of Psychology, 36, 573-61 1.

Schneider, B. (1990). Organizational climate and culture. San Francisco: Jossey-Bass.

Schneider, B., \& Bowen, D. E. (1995). Winning the service game. Cambridge, MA: Harvard Business School Press.

Schneider, B., \& Hall, D. T. (1972). Toward specifying the concept of work climate: A study of Roman Catholic Diocesan priests. Journal of Applied Psychology, 56, 447-455.

Schneider, B., Parkington, J. J., \& Buxton, V. M. (1980). Employee and customer perceptions of 
service in banks. Administrative Science Quarterly, 25, 252-267.

Schneider, B., Salvaggio, A. N., \& Subirats, M. (2002). Climate strength: A new direction for climate research. Journal of Applied Psychology, 87, 220-229.

Schneider, B., White, S. S., \& Paul, M. C. (1998). Linking service climate and customer perceptions of service quality: Test of a causal model. Journal of Applied Psychology, 83, 150163.

Schwab, D. P. (1980). Construct validity in organizational behavior. Research in Organizational Behavior, 2, 3-42.

Sturman, M. C., \& Short, J. C. (2000). Lump-sum bonus satisfaction: Testing the construct validity of a new pay satisfaction dimension. Personnel Psychology, 53, 673-700.

Tannenbaum, S. I. (1997). Enhancing continuous learning: Diagnostic findings from multiple companies. Human Resource Management, 30, 437-452.

Thayer, P. W., \& Teachout, M. S. (1995). A climate for transfer model (Report AL/HR-TP-19950035). Brooks Air Force Base, TX: Air Force Material Command.

Tracey, J. B. (1998, April 25). A three-dimensional model of the transfer of training climate. In W. E. K. Lehman \& M. Cavanaugh (Co-Chairs), Recent trends in the study of transfer climate: Research, theory, and consultation. Symposium presented at the annual meeting of the Society for Industrial and Organizational Psychology, Dallas, TX.

Tracey, J. B., Hinkin, T. R., Tannenbaum, S. I., \& Mathieu, J. E. (2001). The influence of individual characteristics and the work environment on varying levels of training outcomes. Human Resources Development Quarterly, 15, 5-24.

Tracey, J. B., Tannenbaum S. I., \& Kavanagh, M. J. (1995). Applying trained skills on the job: The importance of the work environment. Journal of Applied Psychology, 80, 239-252. 
Youndt, M. A., Snell, S. A., Dean, J. W., \& Lepak, D. P. (1996). Human resource management, manufacturing strategy, and firm performance. Academy of Management Journal, 39, 836866.

Zohar, D. (1980). Safety climate in industrial organizations: Theoretical and applied implications. Journal of Applied Psychology, 65, 96-102. 


\section{APPENDIX}

\section{Items for the General Training Climate Scale (GTCS)}

Managerial Support (MS):

1. Supervisors give recognition and credit to those who apply new knowledge and skills to their work.

2. Supervisors match associates' needs for personal and professional development with opportunities to attend training.

3. Independent and innovative thinking are encouraged by supervisors.

4. Top management expects high levels of performance at all times.

5. Top management expects continuing technical excellence and competence.

Job Support (JS):

1. Gaining new information about ways to perform work more effectively is important in this organization.

2. Job assignments are designed to promote personal development.

3. Learning new ways of performing work is valued in this organization.

4. Work assignments include opportunities to learn new techniques and procedures for improving performance.

5. There is a strong belief that continuous learning is important to successful job performance. Organizational Support (OS):

1. There is a performance appraisal system that ties financial rewards to use of newly acquired knowledge and skills.

2. This organization offers excellent training programs.

3. Employees are provided with resources necessary to acquire and use new knowledge and 
skills.

4. There are rewards and incentives for acquiring and using new knowledge and skills in one's job.

5. This organization rewards employees for using newly acquired knowledge and skills on the job.

Note. All items were evaluated using a 5-point rating scale, 1 (strongly disagree) to 5 (strongly agree). 